\title{
How to Best Detect Portal Vein Tumor Thrombosis in Patients with Hepatocellular Carcinoma Meeting the Milan Criteria: Gadoxetic Acid-Enhanced MRI versus Contrast-Enhanced CT
}

\author{
Jae Seok Bae ${ }^{a, b}$ Jeong Min Lee ${ }^{a-c}$ Jeong Hee Yoon ${ }^{a, b}$ Siwon Jang ${ }^{d}$ \\ Jin Wook Chung ${ }^{a-c}$ Kyung Bun Lee ${ }^{e} \quad$ Nam-Joon $\mathrm{Yi}^{f}$ Jeong-Hoon Lee ${ }^{g}$ \\ a Department of Radiology, Seoul National University Hospital, Seoul, Republic of Korea; \\ ${ }^{b}$ Department of Radiology, Seoul National University College of Medicine, Seoul, Republic \\ of Korea; ' Institute of Radiation Medicine, Seoul National University Medical Research \\ Center, Seoul, Republic of Korea; ${ }^{d}$ Department of Radiology, SMG-SNU Boramae Medical \\ Center, Seoul, Republic of Korea; ${ }^{e}$ Department of Pathology, Seoul National University \\ Hospital, Seoul, Republic of Korea; ${ }^{f}$ Department of Surgery, Seoul National University \\ Hospital, Seoul, Republic of Korea; 9 Department of Internal Medicine, Seoul National \\ University Hospital, Seoul, Republic of Korea
}

\section{Keywords}

Hepatocellular carcinoma - Portal vein thrombosis - Gadoxetic acid-enhanced MRI . Contrast-enhanced CT

\begin{abstract}
Introduction: The detection of portal vein tumor thrombosis (PVTT) in patients with hepatocellular carcinoma (HCC) meeting the Milan criteria is of critical importance as PVTT is known to be a contraindication to transplantation and an indicator of a dismal prognosis. Objective: To determine which modality may best detect PVTT, we compared the diagnostic performance of gadoxetic acid-enhanced MRI (GA-MRI) and contrast-enhanced CT (CECT) in HCC patients meeting the Milan criteria. Methods: We retrospectively enrolled 310 patients with HCCs meeting the Milan criteria who underwent both GA-MRI and CECT between June 2007 and May 2017. Among them, 44 patients were demonstrated to have PVTT while 266 patients had no PVTT. Two radiologists then assessed GA-MRI and CECT images for the presence of PVTT on a 5-point scale as well as vessel expansion, continuity with tumors, and enhancement on both modalities, as well as T2 hyperintensity and diffusion restriction on GA-MRI. The McNemar test was used to compare sensitivity and specificity of GA-MRI and CECT for the detection of PVTT, and Fisher's exact test was used to compare their imaging features.
\end{abstract}


Results: GA-MRI showed higher sensitivity values than CECT in detecting PVTT (reviewer 1, 93.2\% [41/44] vs. 77.3\% [34/44]; reviewer 2, 88.6\% [39/44] vs. 70.5\% [31/44]) (both $p=0.039)$. Specificity of GA-MRI and CECT demonstrated no difference (reviewer 1, 95.5\% [254/266] vs. 95.1\% [253/266]; reviewer 2, 97.0\% [258/266] vs. 97.4\% [259/266]) (both $p>0.999$ ). Continuity with tumors and enhancement were more frequently observed on GA-MRI than on CECT ( $p<0.050$, both reviewers). Conclusion: GA-MRI detected PVTT more often than CECT in HCC patients meeting the Milan criteria and better depicted PVTT in continuity with tumors and those showing enhancement than CECT.

\section{Introduction}

Portal vein tumor thrombosis (PVTT), which typically arises from the invasion of hepatocellular carcinoma (HCC) into the portal vein of patients with liver cirrhosis [1], can be found in up to $23.5-34.1 \%$ of HCC patients [2, 3]. Unfortunately, curative surgical treatments such as transplantation or surgical resection are contraindicated in the presence of PVTT, and thus the prognosis of patients with this disease has been shown to be dismal [4,5]. In contrast, bland portal vein thrombosis (PVT), which is attributed to sluggish portal venous flow in cirrhotic patients with portal hypertension [6], is considered to be treatable via anticoagulation and/or thrombolytic treatment [7], and patients with bland PVT can undergo liver transplantation [8]. Hence, in determining the therapeutic strategy and in predicting patient survival, the detection of non-bland PVTT is of paramount clinical importance, particularly for HCC patients meeting the Milan criteria who are potential candidates for liver transplantation [9].

Until now, first-line diagnostic tests for the evaluation of nodules in patients with liver cirrhosis or in those suspected of having HCC have been dynamic contrast-enhanced CT (CECT) or dynamic MRI using extracellular gadolinium contrast media [10-13]. Recently, however, several studies have demonstrated that gadoxetic acid-enhanced MRI (GA-MRI) may provide higher sensitivity for HCC detection than CECT or MRI using extracellular gadolinium contrast media owing to the improved lesion-to-liver contrast at hepatobiliary phase imaging [14-20]. Consequently, many major guidelines, including those of the European Association for the Study of the Liver, American Association for the Study of Liver Diseases, Liver Imaging Reporting and Data System, and the Asian-Pacific Association for the Study of the Liver, now include GA-MRI as a first-line diagnostic test $[11-13,21]$.

Yet, in contrast to that for HCC, GA-MRI may have a potential disadvantage in detecting PVTT owing to the decreased contrast between the portal vein and liver parenchyma during the dynamic phase, resulting from the early GA uptake, which begins approximately 1 min after contrast agent administration [22, 23]. Moreover, as GA has only $25 \%$ of the gadolinium molecules that extracellular gadolinium contrast media have, the resultant weaker contrast enhancement may also potentially hinder the detection of small enhancing lesions [24]. Although a previous study had demonstrated that GA-MRI has a sensitivity ranging between 81 and 93\% [25], CECT was used as a reference standard in that study, which precluded the comparison of both techniques in detecting PVTT. Similarly, other studies that have reported a sensitivity of CECT ranging between 48 and $86 \%$ in detecting PVTT did not compare it to the sensitivity of GA-MRI, let alone in the same individual [26, 27]. Therefore, the purpose of our study was to intraindividually compare the diagnostic performance of GA-MRI to that of CECT in PVTT detection in HCC patients meeting the Milan criteria.

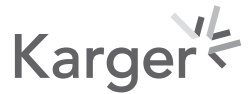


Excluded patients $(n=2,439)$

- Had undergone previous treatment before surgery or TACE $(n=2,254)$

- Time interval between GA-MRI and CECT $>1$ month $(n=145)$

- Time interval between GA-MRI or CECT and surgery $>2$ months $(n=17)$

- Time interval between GA-MRI or CECT and TACE $>2$ months $(n=23)$

Excluded patients $(n=214)$ - With microscopic PVT

Patients without macroscopic PVT $(n=414)$

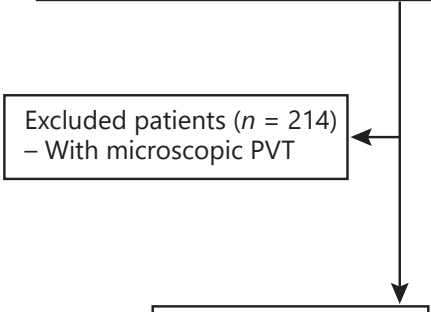

Patients without PVT $(n=200)$
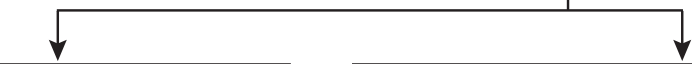

Patients without pathological information $(n=112)$

Patients with angiography or cone beam CT Patients with post-TACE CT

Fig. 1. Flow diagram of the study population. GA-MRI, gadoxetic acid-enhanced magnetic resonance imaging; CECT, contrast-enhanced computed tomography; TACE, transarterial chemoembolization; PVT, portal vein thrombosis; PVTT, portal vein tumor thrombosis.

\section{Materials and Methods}

\section{Patients}

This retrospective study was approved by our institutional review board for a chart review, and the requirement for informed consent was waived. From the radiological and pathological databases of our hospital, we found 3,042 HCC patients meeting the Milan criteria who had undergone both multidetector CECT and GA (Primovist or Eovist; Bayer Healthcare, Berlin, Germany)-enhanced MRI between June 2007 and May 2017. HCCs were confirmed at pathological examination after surgery or were noninvasively diagnosed according to American Association for the Study of Liver Diseases guidelines [12]. Among them, we excluded patients whose (a) treatment such as portal vein embolization was performed prior to surgery or transarterial chemoembolization (TACE), (b) the time interval between GA-MRI and CECT exceeded 1 month, or (c) the time interval between CECT or GA-MRI and surgery or TACE exceeded 2 months. In 491 patients with pathologically confirmed HCC, the status of the portal vein was assessed. Seventy-seven of these patients were pathologically diagnosed to have macroscopic PVTs (66 patients with bland PVT and 11 patients with PVTT) and underwent surgical resection for HCC (35 patients) or liver transplantation (42 patients). Among the 42 patients who were subject to liver transplantation, 41 patients had bland PVT, and 1 patient had PVTT. In the remaining 414 patients in whom macroscopic PVT was not histopathologically confirmed, we only included 200 patients who were also pathologically confirmed not to have microscopic PVT (Fig. 1).

In 112 patients without pathologic evidence, a radiologic diagnosis of HCC was made according to the noninvasive diagnostic criteria of the American Association for the Study of Liver Diseases guidelines based on dynamic CECT and/or GA-MRI performed within 2 months (range, 0-53 days) prior to TACE. Among these

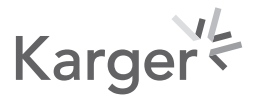


patients, we only included 33 patients who showed PVTT on angiography or cone beam CT images obtained during TACE and non-contrast CT. Other patients were excluded for the following reasons: (a) no visible PVT during TACE, (b) TACE was performed using drug-eluting beads rather than iodized oil, and (c) there was indeterminate iodized oil uptake at post-TACE non-contrast CT.

Therefore, in total, there were 11 patients with pathologically proven PVTT, 33 patients with radiologically proven PVTT, 66 patients with bland PVT, and 200 patients without macroscopic or microscopic PVT. The mean time interval between CECT and GA-MRI was 10.8 days \pm 9.7 . GA-MRI was performed later $(n=$ $212)$ or earlier $(n=40)$ than CECT, or it was performed on the same day as CECT $(n=58)$. It should be noted, however, that among the 310 patients included in the final study population, 49 patients had been enrolled in our previous study, which included 22 patients with PVTT and 27 patients without PVTT [25]. However, the diagnostic performance of CECT for the detection of PVTT was not assessed in the aforementioned prior study, and the former study comprised less than one-sixth of the current study population.

\section{Reference Standard for the Diagnosis and Characterization of PVT}

Pathological Diagnosis

One experienced pathologist (K.B.L.: 16 years of experience in liver disease) who was provided the clinical information and imaging results of the 277 pathologically confirmed study patients reviewed the surgical specimens and confirmed the presence and type of PVT. In the case of liver transplantation, the explanted liver was initially cut into 5 -mm-thick sections in the sagittal plane. For small $(<5 \mathrm{~mm})$ lesions, thinner (1-mm) sections were obtained, and lesion-by-lesion correlations between pathological specimens and GA-MRI images were performed. When the exact PVT location was not mentioned in the pathological reports, the operative record was used to confirm its location. A thrombus was categorized as PVTT if viable tumor cells were present. Thereafter, PVTT was classified as either macroscopic or microscopic. We excluded microscopic PVTT as it cannot be depicted on current imaging modalities. Thus, only macroscopic PVTT was included. A thrombus was considered to be bland if it contained fibrin and/or blood clots but no viable tumor cells. If PVTT and bland PVT coexisted in the same patient, the patient was regarded as having PVTT. Both PVTT and bland PVT were classified as either complete or partial PVT, and as major branch or segmental branch PVT.

\section{Radiological Diagnosis}

In the remaining 33 patients (10.6\%; total: 310 ) without pathological confirmation, we used radiological diagnostic criteria based on the image findings obtained during and after TACE. The detailed procedure of TACE using iodized oil (Lipiodol; Andre Guerbet, Aulnay-sous-Bios, France) has been described previously [28]. During TACE, angiography and/or cone beam CT was performed to localize the tumor and to find feeding vessels to the tumor. Two weeks after TACE, non-contrast-enhanced CT was routinely performed to assess therapeutic efficacy. Because CT and/or MRI were subjects of our study, we exploited the imaging findings based on these studies instead of other noninvasive diagnostic criteria. PVTT was diagnosed if both of the following criteria were met: (a) thin linear or chain-like opacification in the portal vein at angiography ("thread-and-streak sign") or cone beam CT after injection of contrast material during TACE [29] and (b) uptake of iodized oil of the thrombus was demonstrated on the follow-up CT after TACE [30]. One boardcertified radiologist (J.S.B.: 6 years of experience in abdominal imaging), who did not participate in the image analysis, reviewed the post-TACE CT images and excluded indeterminate iodized oil uptakes.

\section{Imaging Techniques}

Dynamic CECT

CECT examinations, including unenhanced, arterial, portal venous, and delayed phases, were obtained. Details of our liver CT protocol and parameters are described in the online supplementary Material and Table S1 (for all online suppl. material, see www.karger.com/di/10.1159/000505191).

\section{GA-MRI}

GA-MRI scans were obtained using either a 3.0- or 1.5-T system. The liver MRI protocol used in our hospital included a breath-hold fat-saturated T2-weighted fast spin echo or turbo spin echo sequence, a three-dimensional T1-weighted dual echo sequence, diffusion-weighted imaging, and dynamic three-dimensional fat-saturated T1-weighted gradient echo sequences, including hepatobiliary phase images. Detailed information of our GA-MRI examinations including its parameters is described in the online supplementary Material and Table S2. 
Bae et al.: Detecting Portal Vein Tumor Thrombosis: MRI versus CT

\section{Image Analysis}

Two board-certified radiologists (J.H.Y. and S.J: 12 and 5 years of experience in abdominal imaging, respectively) independently evaluated all GA-MRI and CECT images for the presence and characteristics of PVTT on a picture-archiving and communication system (Infinitt PACS Viewer; Infinitt, Seoul, Republic of Korea). The reviewers were informed that the patients had HCC but were blinded to all other clinical and histopathological information. The reviewers graded the presence of PVTT on a 5-point scale according to their degree of confidence: (1) definitely absent (0-20\%); (2) probably absent (21-40\%); (3) indeterminate (41-60\%); (4) probably present (61-80\%); and (5) definitely present (81-100\%). For example, when unequivocal-enhancing soft tissue is identified in the portal vein, it was scored as 5 , whereas occlusion or an obscured vein in contiguity with HCC without unequivocal enhancement was scored as 3 ; in the case of a PVTT presence score $\geq 3$, the location of the suspected PVTT was recorded, and the presence of the following imaging findings, regarded as the characteristic imaging features of PVTT, were evaluated: (a) enhancement of the PVT after administration of contrast material compared to precontrast images $(\geq 20 \mathrm{HU}$ on CT and $\geq 15 \%$ on GA-MRI) [31, 32]; (b) expansion of the involved portal vein (diameter $\geq 1.8 \mathrm{~cm}$ for the main or left portal vein, $\geq 1.6 \mathrm{~cm}$ for the right portal vein, and disproportionate enlargement when compared with non-affected same-order portal vein branches in the same lobe) [33, 34]; and (c) continuity of the PVT with the HCC [35]. All available images of each modality were utilized for the assessment of the above imaging features. On GA-MRI, the following additional features were assessed: (a) T2-weighted hyperintensity of the PVT [25] and (b) restricted diffusion within the PVT defined as an increased signal on diffusionweighted images and a decreased signal on apparent diffusion coefficient maps [25, 33]. In order to determine diffusion restriction, diffusion-weighted images obtained using a $b$ value of at least $500 \mathrm{~s} / \mathrm{mm}^{2}$ were used.

\section{Statistical Analysis}

The clinical characteristics of patients with and without PVTT were compared using the $\chi^{2}$ test for categorical variables and the independent $t$ test for numerical variables. To determine the sensitivity and specificity of CECT and GA-MRI in the detection of PVTT, we regarded the PVTT presence score of 3 or greater as positive. The McNemar test was used to compare the sensitivity and specificity of GA-MRI and CECT for the detection of PVTT. In addition, we conducted further subgroup analyses on the basis of the PVTT location (major branch or segmental branch), PVTT extent (complete or partial), underlying liver condition (presence or absence of liver cirrhosis), Child-Pugh class (A or B/C), and the reference standards (pathology or imaging). The impact of magnetic field strength on the assessment of PVTT was evaluated as well. Agreement between the reviewers for the detection of PVTT and imaging features of PVTT were assessed using linearly weighted $\kappa$ statistics, and the following conventional interpretation was used: $\kappa=0-0.20$, poor agreement; $\kappa=0.21-$ 0.40 , fair agreement; $\kappa=0.41-0.60$, moderate agreement; $\kappa=0.61-0.80$, substantial agreement; and $\kappa=$ 0.81-1.00, almost perfect agreement. All statistical analyses were performed using commercially available software (MedCalc, version 14.8.1; MedCalc Software, Mariakerke, Belgium; SPSS, version 23.0; SPSS, Chicago, IL, USA, and SAS, version 9.4; SAS Institute Inc., Cary, NC, USA). A two-sided $p$ value $<0.050$ was considered to indicate a statistically significant difference.

\section{Results}

\section{Patient Characteristics}

The clinical and histopathological characteristics of the 310 study patients (overall mean age \pm SD: $56.9 \pm 9.2$ years; 235 men [mean age \pm SD: $57.4 \pm 8.4$ years] and 75 women [mean age \pm SD: $55.5 \pm 11.3$ years]) are shown in Table 1 . Preoperative serum levels of $\alpha$-fetoprotein and protein induced by vitamin $\mathrm{K}$ absence or antagonist II were shown to be significantly higher in the group with PVTT than in those without PVTT ( $p<0.001$ for both). More patients were of Child-Pugh class A among patients with PVTT $(p=0.046)$. There were no differences in the causes of HCC, presence of liver cirrhosis, or in any tumor characteristic, including tumor multiplicity and maximal tumor diameter, between those with and without PVTT. Further description of PVT distribution is demonstrated in online supplementary Figure S1.

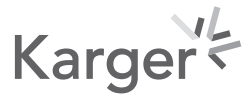


Table 1. Patient characteristics

\begin{tabular}{|c|c|c|c|c|}
\hline \multirow[t]{2}{*}{ Characteristics } & \multirow{2}{*}{$\begin{array}{l}\text { With PVTT } \\
(n=44)^{*}\end{array}$} & \multicolumn{2}{|c|}{ Without PVTT $(n=266)$} & \multirow[t]{2}{*}{$p$ value } \\
\hline & & $\begin{array}{l}\text { with benign PVT } \\
(n=66)\end{array}$ & $\begin{array}{l}\text { without PVT } \\
(n=200)\end{array}$ & \\
\hline Age, years & $56.5 \pm 10.5$ & $56.1 \pm 8.4$ & $57.3 \pm 9.2$ & 0.755 \\
\hline Male:female ratio & $35: 9$ & $49: 17$ & $151: 49$ & 0.533 \\
\hline \multicolumn{5}{|l|}{ Time interval, days } \\
\hline Between GA-MRI and CT & $9.5(0-31)$ & $1.0(0-31)$ & $12.0(0-31)$ & 0.118 \\
\hline Between GA-MRI/CT and surgery/TACE & $21.5(3-52)$ & $18.0(0-56)$ & $22.0(0-62)$ & 0.813 \\
\hline \multicolumn{5}{|l|}{ Serum level of tumor markers } \\
\hline$\alpha$-Fetoprotein, ng/mL & $36.5(2.1-304,700)$ & $10.2(1.0-137,200)$ & $8.0(1.0-124,000)$ & $<0.001$ \\
\hline PIVKA-II, mAU/mL & $114.5(2.0-30,320)$ & $28.0(6.0-48,000)$ & $33.0(10.0-13,520)$ & $<0.001$ \\
\hline Cause of HCC & & & & 0.276 \\
\hline Hepatitis B virus & $34(77)$ & $49(74)$ & $166(83)$ & \\
\hline Hepatitis $C$ virus & $4(9)$ & $7(11)$ & $15(8)$ & \\
\hline Alcohol & $4(9)$ & $9(14)$ & $10(5)$ & \\
\hline Unknown & $2(5)$ & $1(1)$ & $9(4)$ & \\
\hline Liver cirrhosis & $34(77)$ & $64(97)$ & $153(77)$ & 0.501 \\
\hline Child-Pugh class & & & & 0.046 \\
\hline $\mathrm{A}$ & $34(77)$ & $21(32)$ & $143(72)$ & \\
\hline $\mathrm{B}$ or $\mathrm{C}$ & $10(23)$ & $45(68)$ & $57(28)$ & \\
\hline Infiltrative HCC & $0(0)$ & $0(0)$ & $2(1)$ & $>0.999$ \\
\hline Tumor multiplicity & & & & 0.085 \\
\hline Single tumor & $28(64)$ & $36(55)$ & $166(83)$ & \\
\hline Multiple tumors & $16(36)$ & $30(45)$ & $34(17)$ & \\
\hline Maximal tumor diameter, $\mathrm{cm}$ & & & & 0.117 \\
\hline Mean \pm SD & $2.78 \pm 0.96$ & $2.02 \pm 0.83$ & $2.68 \pm 1.05$ & \\
\hline Range & $1.4-5.0$ & $1.0-4.5$ & $1.0-5.0$ & \\
\hline
\end{tabular}

Data are numbers $(\%)$ of patients, means \pm SD, or medians (ranges). PVTT, portal vein tumor thrombosis; TACE, transarterial chemoembolization; PIVKA-II, protein induced by vitamin K absence or antagonist-II; AU, arbitrary units; HCC, hepatocellular carcinoma. $p$ values were calculated between groups with and without PVTT. HCC diagnosis was based on pathological findings.

\section{Diagnostic Performance of GA-MRI and CECT for PVTT}

Detection of PVTT

GA-MRI demonstrated significantly higher sensitivity than CECT in the detection of PVTT (reviewer 1,93.2\% [41/44] vs. 77.3\% [34/44]; reviewer 2, 88.6\% [39/44] vs. 70.5\% [31/44]) ( $p=0.039$ for both) (Fig. 2; Table 2). In terms of specificity for the detection of PVTT, however, there were no significant differences between GA-MRI and CECT (reviewer 1, 95.5\% [254/266] vs. $95.1 \%$ [253/266]; reviewer 2, 97.0\% [258/266] vs. 97.4\% [259/266]) ( $p>0.999$ for both). In addition, the positive predictive value for the detection of PVTT was higher on GA-MRI than CECT (reviewer 1, 77.4\% [41/53] vs. 72.3\% [34/47]; reviewer 2, 83.0\% [39/47] vs. 81.6\% [31/38]). The negative predictive value for the detection of PVTT was also higher on GA-MRI than on CECT (reviewer 1, 98.8\% [254/257] vs. 96.2\% [253/263]; reviewer 2, $98.1 \%$ [258/263] vs. 95.2\% [259/272]). In terms of interobserver agreement, GA-MRI showed nearly perfect agreement $(\kappa=0.890)$ while that of CECT was substantial $(\kappa=0.742)$. Intraobserver agreements between GA-MRI and CECT were almost perfect or substantial in both reviewers ( $\kappa=0.816$ and 0.798 for reviewers 1 and 2, respectively).

Subgroup Analysis

GA-MRI was also shown to be superior to CECT in the detection of PVTT under the following conditions: patients with liver cirrhosis (reviewer 1, 97.1\% [33/34] vs. 76.5\% 


\begin{tabular}{l|l}
\hline \multicolumn{2}{l}{ Liver Cancer 2020;9:293-307 } \\
\hline DOI: 10.1159/000505191 & $\begin{array}{l}\text { @ 2020 The Author(s). Published by S. Karger AG, Basel } \\
\text { www.karger.com/lic }\end{array}$ \\
\hline
\end{tabular}

\section{Liver Cancer}

Bae et al.: Detecting Portal Vein Tumor Thrombosis: MRI versus CT

Table 2. Comparison of diagnostic performance between GA-MRI and CECT in the detection of PVTT

\begin{tabular}{|c|c|c|c|c|c|c|c|c|}
\hline & \multicolumn{4}{|l|}{ Sensitivity, \% } & \multicolumn{4}{|l|}{ Specificity, \% } \\
\hline & GA-MRI & CECT & difference $(95 \% \mathrm{CI})$ & $p$ value & GA-MRI & CECT & difference $(95 \%$ CI) & $p$ value \\
\hline Reviewer 1 & $93.2(41 / 44)$ & $77.3(34 / 44)$ & $15.9(3.4$ to 28.4$)$ & 0.039 & $95.5(254 / 266)$ & $95.1(253 / 266)$ & $0.4(-3.0$ to 2.3$)$ & $>0.999$ \\
\hline Reviewer 2 & $88.6(39 / 44)$ & $70.5(31 / 44)$ & 18.1 (3.7 to 32.7 ) & 0.039 & $97.0(258 / 266)$ & $97.4(259 / 266)$ & $-0.4(-2.0$ to 1.3$)$ & $>0.999$ \\
\hline
\end{tabular}

Except where indicated, data are percentages, with numerators and denominators in parentheses. GA-MRI, gadoxetic acid-enhanced MRI; CECT, contrastenhanced CT; PVTT, portal vein tumor thrombosis. Differences refer to the value of CECT subtracted from that of GA-MRI.
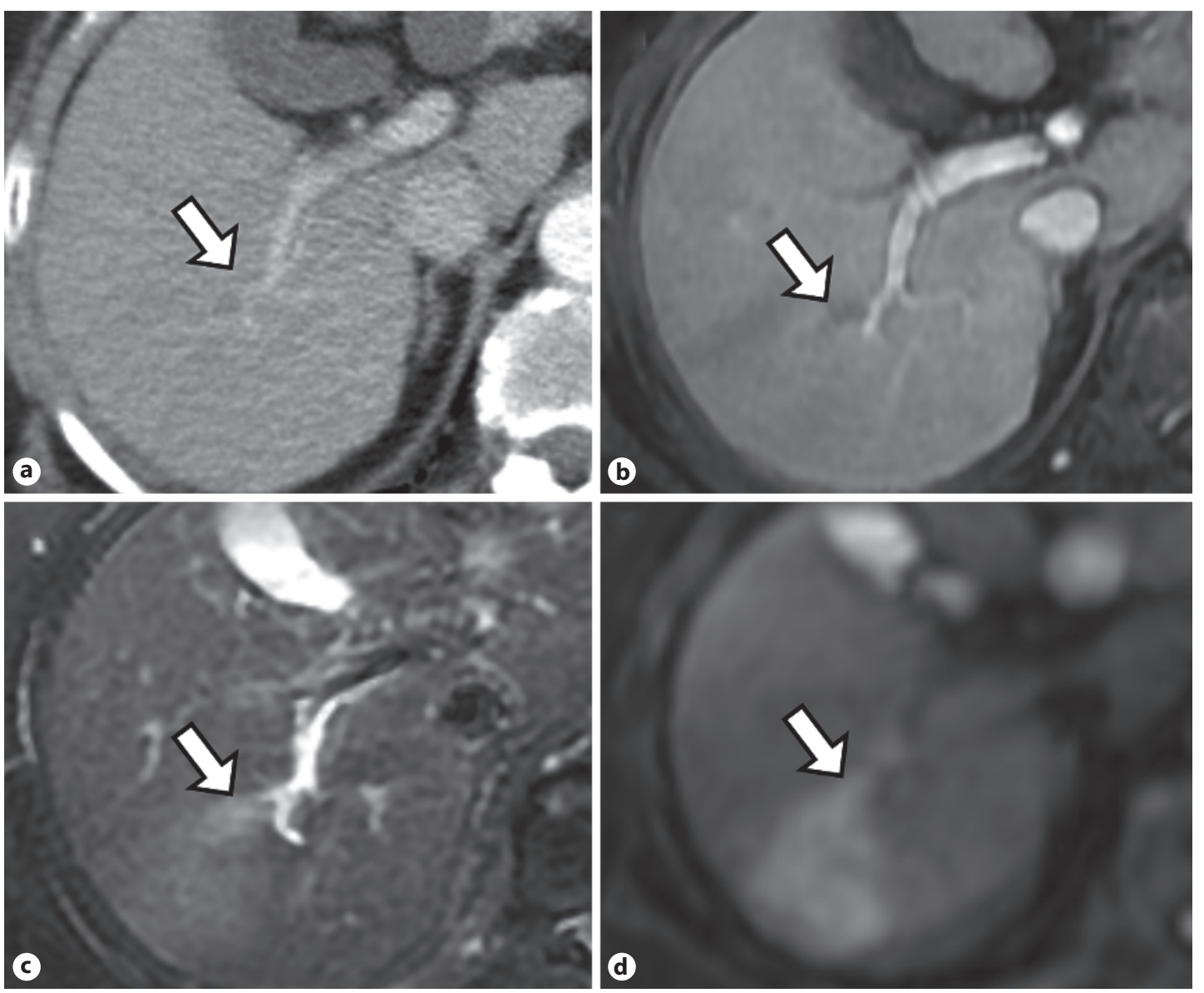

Fig. 2. Contrast-enhanced computed tomography and gadoxetic acid-enhanced magnetic resonance imaging (GA-MRI) scans of a 63-year-old woman with hepatocellular carcinoma and segmental portal vein tumor thrombosis (PVTT). a Axial portal venous phase CT image shows a suspicious segmental PVTT (arrow). Our 2 radiologists graded that the PVTT was probably absent and indeterminate, respectively. b-d GA-MRI performed 6 days after contrast-enhanced CT revealed portal vein thrombosis (arrow) was hypointense on postcontrast T1-weighted images in the portal venous phase (arrow) (b), and hyperintense on the axial T2weighted (c) and diffusion-weighted images obtained with a $b$ value of $800 \mathrm{~s} / \mathrm{mm}^{2}$ (d). Our 2 radiologists assessed that PVTT was definitely present and indeterminate, respectively. This patient underwent transarterial chemoembolization, and compact iodized oil uptake at the PVTT was demonstrated. 
Bae et al.: Detecting Portal Vein Tumor Thrombosis: MRI versus CT

Table 4. Comparison of imaging features of PVTT on GA-MRI and CECT

\begin{tabular}{|c|c|c|c|c|c|c|c|c|}
\hline \multirow[t]{2}{*}{ Imaging features } & \multicolumn{3}{|l|}{ Reviewer 1} & \multicolumn{3}{|l|}{ Reviewer 2} & \multicolumn{2}{|c|}{ Interobserver agreement } \\
\hline & GA-MRI & CECT & $p$ value & GA-MRI & CECT & $p$ value & GA-MRI & CECT \\
\hline Vessel expansion & $90.9(40 / 44)$ & $81.8(36 / 44)$ & 0.015 & $77.3(34 / 44)$ & $61.4(27 / 44)$ & 0.150 & $0.249(32 / 41)$ & $0.152(26 / 32)$ \\
\hline Continuity with tumor & $95.5(42 / 44)$ & $79.5(35 / 44)$ & 0.038 & $88.6(39 / 44)$ & $68.2(30 / 44)$ & 0.029 & $0.192(33 / 41)$ & $0.375(27 / 32)$ \\
\hline Enhancement & $86.4(38 / 44)$ & $84.1(37 / 44)$ & $<0.001$ & $79.5(35 / 44)$ & $68.2(30 / 44)$ & 0.019 & $0.446(35 / 41)$ & $<0.001(27 / 32)$ \\
\hline T2 hyperintensity & $84.1(37 / 44)$ & & & $84.1(37 / 44)$ & & & $0.239(32 / 41)$ & \\
\hline Diffusion restriction & $89.7(35 / 39)$ & & & $89.7(35 / 39)$ & & & $0.164(29 / 37)$ & \\
\hline
\end{tabular}

Data are percentages, with numerators and denominators in parentheses. PVTT, portal vein tumor thrombosis; GA-MRI, gadoxetic acid-enhanced MRI; CECT, contrast-enhanced CT. Numbers in parentheses are the number of actual agreements divided by the total number.

[26/34], $p=0.016$ ) and patients with Child-Pugh class A (reviewer 2, 94.1\% [32/34] vs. $67.6 \%$ [23/34], $\mathrm{p}=0.004$ ) (Table 3). The impact of magnetic field strength on the detection of PVTT is described in online supplementary Table S3.

Comparison of the Imaging Findings of PVTT between GA-MRI and CECT

The characteristic imaging findings of PVTT, including vessel expansion, continuity with the tumor, and enhancement for both GA-MRI and CECT, as well as T2 hyperintensity and restricted diffusion were common in PVTT (Table 4). Among the 3 imaging features common to GA-MRI and CECT, continuity with the tumor and enhancement were more frequently observed on GA-MRI than on CECT according to both reviewers (reviewer 1, 95.5\% [42/44] vs. $79.5 \%$ [35/44], $p=0.038$; reviewer 2 , 88.6\% [39/44] vs. $68.2 \%$ [30/44], $p=0.029$, for continuity with tumors; and reviewer $1,86.4 \%$ [38/44] vs. 84.1\% [37/44], $p<0.001$; reviewer $2,79.5 \%$ [35/44] vs. $68.2 \%$ [30/44], $p=0.019$ for enhancement). Vessel expansion was more frequently observed on GA-MRI than CECT according to 1 reviewer (reviewer 1,90.9\% [40/44] vs. 81.8\% [36/44], $p=0.015$ ) (Fig. 3). The impact of magnetic field strength on the imaging features of PVTT is described in online supplementary Table S4.

Among the 8 patients in whom PVTT was only detected on GA-MRI but not on CECT by reviewer 1, those 3 imaging features were more commonly demonstrated on GA-MRI than on CECT: continuity with the tumor $(100 \%$ [8/8] vs. $25 \%$ [2/8]), enhancement (50\% [4/8] vs. $25 \%$ [2/8]), and vessel expansion (75\% [6/8] vs. 12.5\% [1/8]) (Fig. 4). As for diffusion restriction and T2 hyperintensity, they were shown in $100 \%$ (8/8) and $87.5 \%$ (7/8) of PVTT, respectively, according to reviewer 1 . In reviewer 2 , among the 10 PVTTs that were only detected on GA-MRI but not on CECT, those 3 imaging features were also more commonly demonstrated by GA-MRI than by CECT: continuity with the tumor $(100 \%$ [10/10] vs. $0 \%$ $[0 / 10])$, enhancement (70\% [7/10] vs. $0 \%$ [0/10]), and vessel expansion $(70 \%$ [7/10] vs. 0\% $[0 / 10])$. Both diffusion restriction and T2 hyperintensity were detected in $100 \%(10 / 10)$ of PVTT according to reviewer 2 .

\section{Discussion}

Contrary to our initial concerns that GA-MRI may have a theoretical disadvantage in detecting PVTT owing to the decreased contrast between the portal vein and liver parenchyma arising from the early hepatocyte uptake of GA, as well as its lower gadolinium content $[22,24,25]$, our study results demonstrated that GA-MRI better detected PVTT than CECT in HCC patients meeting the Milan criteria ( $p=0.039$ for both reviewers). This is in concordance with previous studies which reported a sensitivity of $81-93 \%$ using GA-MRI for the detection

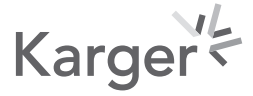



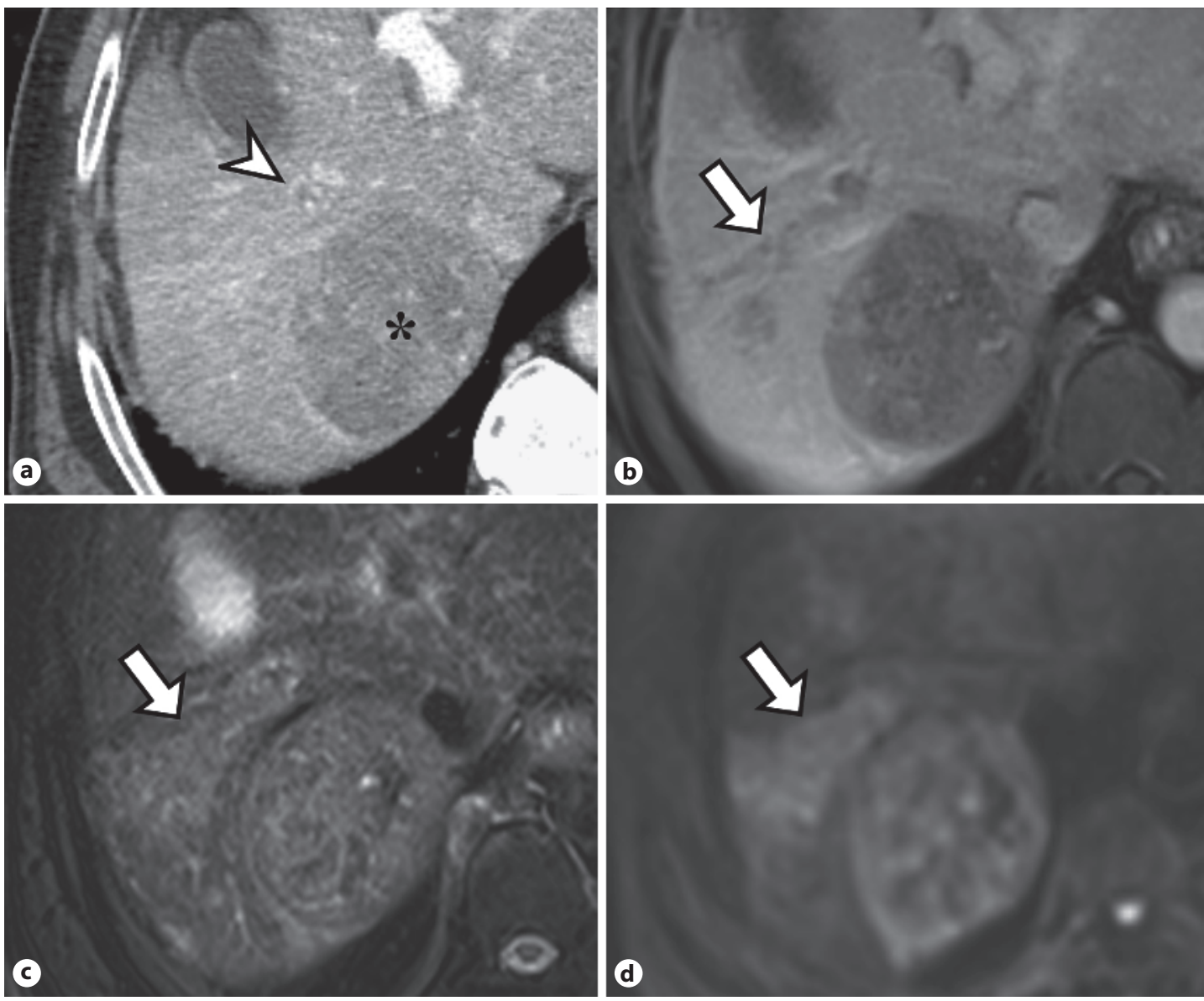

Fig. 3. Contrast-enhanced CT and gadoxetic acid-enhanced magnetic resonance imaging (GA-MRI) of a 75-year-old man with hepatocellular carcinoma (HCC) and segmental portal vein tumor thrombosis (PVTT). a Axial portal venous phase CT image shows HCC (asterisk) and a subtle low attenuation at an adjacent portal vein branch (arrowhead). Our 2 radiologists graded that PVTT was definitely absent and indeterminate, respectively. b-d GA-MRI was performed 18 days after contrast-enhanced CT. Hypointensity was observed in the portal vein branch (arrow) on post-contrast T1-weighted image in the portal venous phase (b), which was shown to be hyperintense on the T2-weighted image (c) and on the diffusion-weighted image obtained with a $b$ value of $800 \mathrm{~s} / \mathrm{mm}^{2}$ (d). Our 2 radiologists assessed that PVTT was definitely present and probably present, respectively. This patient had liver cirrhosis and was graded as Child-Pugh class A. Transarterial chemoembolization was performed, and follow-up non-contrast-enhanced CT demonstrated compact iodized oil uptake at the portal vein branch (not shown).

of PVTT [25] and of 48-86\% using CECT [26, 27, 36]. This result may mainly be attributed to the better depiction of common imaging findings, such as continuity with the tumor in the adjacent liver parenchyma, and the additional contribution of diffusion- and T2-weighted imaging, which are not available with CECT. Indeed, T2-weighted imaging and diffusionweighted imaging have already been advocated for the improved diagnostic capability for PVTT $[25,33]$. Therefore, the theoretical disadvantage of GA-MRI for the detection of PVTT, if it existed at all, may have been overcome by the better delineation of "PVTT showing continuity with the tumor in the adjacent liver parenchyma" or by the additional parameters of T2- and diffusion-weighted imaging. Furthermore, previous studies have demonstrated that GA-MRI also better detects small $(\leq 2 \mathrm{~cm})$ HCCs than CECT in patients who underwent surgery for HCC $[18,37]$, presumably due to the excellent lesion-to-liver contrast at hepatobiliary 

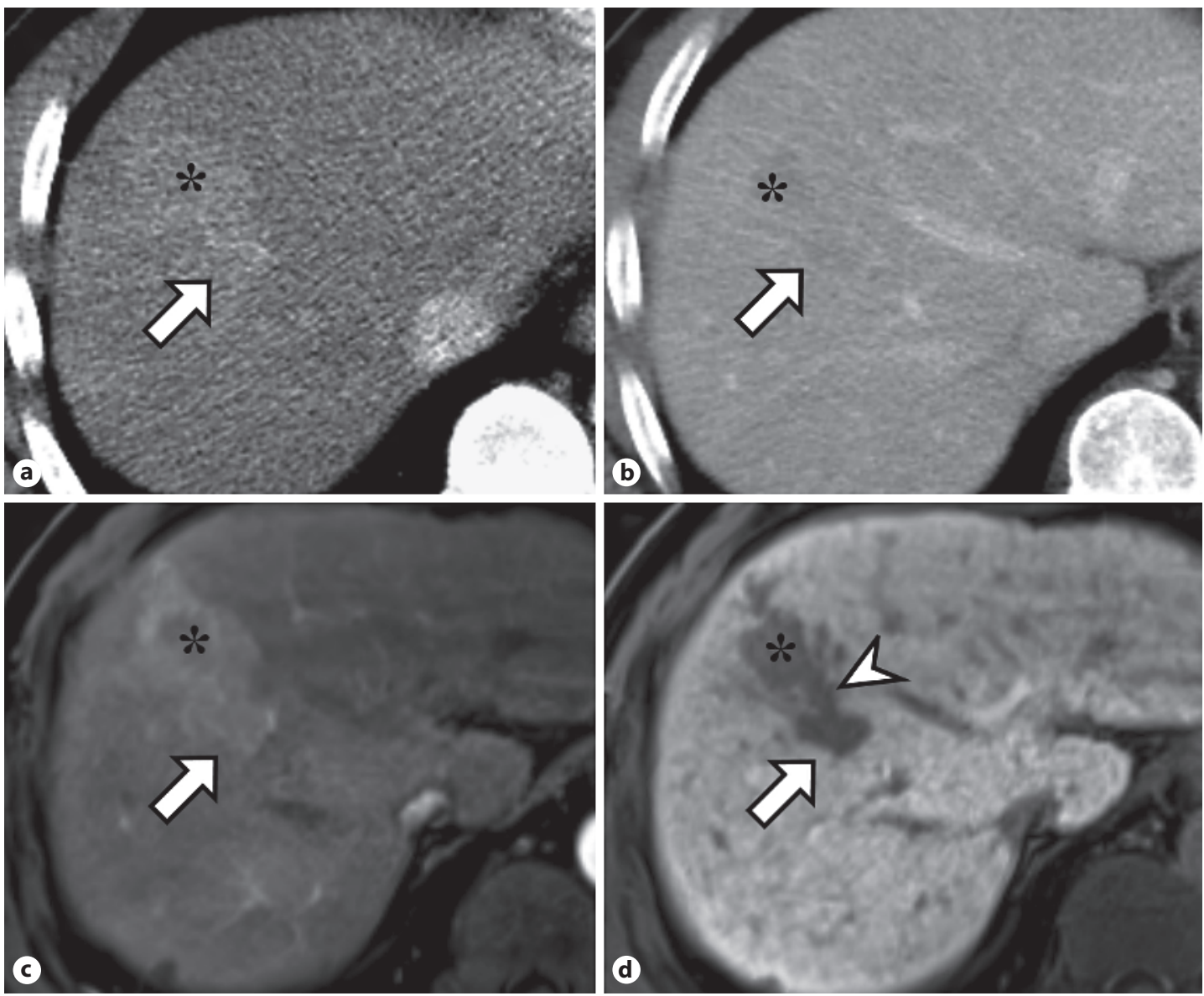

Fig. 4. Contrast-enhanced CT and gadoxetic acid-enhanced MRI (GA-MRI) of a 51-year-old man with hepatocellular carcinoma (HCC) with portal vein tumor thrombosis (PVTT). a, b Axial CT shows HCC (asterisk) with patchy arterial enhancement (a) and subtle portal washout (b). PVTT (arrow) with subtle arterial enhancement and minimal portal washout was observed. This PVTT was detected by only 1 of our reviewers and was graded as 3 (indeterminate). c Axial post-contrast T1-weighted image in the arterial phase shows a patchy-enhancing HCC (asterisk) and an adjacent enhancing PVTT (arrow). d On hepatobiliary phase image, both HCC and PVTT showed hypoenhancement (asterisk and arrow, respectively). PVTT continuity with the HCC was clearly delineated (arrowhead). Transarterial chemoembolization was performed in this patient, and follow-up non-contrast CT demonstrated compact iodized oil uptake at the portal vein branch (not shown).

phase imaging [38]. Considering this high sensitivity of GA-MRI for the detection of PVTT as well as for small HCCs, GA-MRI can additionally be performed in HCC patients meeting the Milan criteria on CECT for more accurate staging and allocation of treatment.

Our subgroup analyses also demonstrated that GA-MRI was superior to CECT in patients with liver cirrhosis (reviewer 1) and in patients with Child-Pugh class A (reviewer 2), although this result was demonstrated by only 1 of the reviewers for each category. In patients with preserved liver function, strong enhancement of the hepatic parenchyma may increase the conspicuity of PVTT and its continuity with the tumor on hepatobiliary phase images $[39,40]$. This may have aided in the detection of PVTT by GA-MRI in patients with Child-Pugh class A compared to patients with Child-Pugh class B or C. Furthermore, although there was no significant difference between GA-MRI and CECT for the detection of PVTT in patients without liver cirrhosis by both reviewers, the small number of patients without liver cirrhosis $(n=10)$ may 
have played a role in its statistical insignificance. In terms of reference standards for PVTT, GA-MRI was superior to CECT with marginal statistical significance (reviewer $1, p=0.070$ ) in the patients with PVTT diagnosed by imaging. This result may also have been caused by the relatively small number of patients with PVTT diagnosed by imaging $(n=33)$, not to mention the even smaller number of patients with pathologically proven PVTT $(n=11)$. Further studies with a larger sample size would be needed to fully evaluate the diagnostic performance of GA-MRI and CECT for the detection of PVTT in patients without liver cirrhosis or in patients with either pathological or radiological reference standards for PVTT. Regarding magnetic field strength, 3.0-T MRI systems have a higher signal-to-noise ratio and contrastto-noise ratio than 1.5-T MRI systems [41]. However, these potential advantages of 3.0-T MRI over 1.5-T MRI were not observed in our study. These results have possibly been caused by the relatively small dose of gadolinium molecules of GA or by more prominent motion artifacts at 3.0-T MRI, although motion artifacts were not assessed in our study [24, 42].

Finally, among the characteristic imaging features of PVTT, continuity with the tumor and enhancement of PVTT were more frequently observed on GA-MRI than on CECT by both reviewers ( $p<0.050$ for all). In addition, 1 of our reviewers assessed that vessel expansion by PVTT was better depicted by GA-MRI than by CECT $(p=0.015)$. Our results are in concordance with previous studies which hinted at a similar tendency [25, 26, 33, 35, 43-45]. This result may be explained by the superior soft tissue contrast of MRI [46], which would result in a better delineation of the continuity between PVTT and HCC, or a more conspicuous outline of the vessels on GA-MRI. In addition, the high signalintensity observed on T2-weighted imaging and restricted diffusion on GA-MRI might have contributed to the good performance of GA-MRI compared to that of CECT. In terms of the interobserver agreement, most imaging features showed relatively low agreements. For instance, the $\kappa$ value for PVTT continuity with the tumor was only 0.192 between both radiologists. We believe that in most instances, this low $\kappa$ value resulted from $\kappa$ paradoxes associated with low $\kappa$ values caused by the effect of prevalence on $\kappa$ in spite of better actual agreement [47]. Indeed, for the PVTT continuity with the tumor, the number of patients showing actual agreements was 33 among 41 patients, which is $>80 \%$. Considering such a high actual agreement, we thought that interobserver agreement might be better than a glance from $\kappa$ values.

There are several limitations to our study that warrant mention. First, as this study is a retrospective single center study, selection bias may have been present. To be specific, the inclusion of patients who underwent both CECT and GA-MRI may have caused selection bias. The choice of the treatment method (e.g., TACE) may have been affected by the results of CT and/or MRI examinations as well. Second, various MRI and CT machines were used for the GA-MRI and CECT examinations assessed in this study owing to its retrospective nature. Indeed, the different textures of images obtained from multiple vendors may have influenced the interpretation of the GA-MRI and CECT findings. However, this heterogeneity present in our study may actually better represent actual routine clinical practice in which various MRI and CT machines are used. Third, exact lesion-by-lesion matching between the surgical specimens and GA-MRI or CECT images was challenging as the pathological planes (sagittal) differed from the imaging planes (transverse). Fourth, as patients with microscopic PVTT were not included in our study, sensitivity for the detection of PVTT may have been exaggerated. However, currently, microscopic PVTT cannot be depicted using any imaging technology, including GA-MRI and CECT, and thus we believe that the exclusion of microscopic PVTT had little impact on the comparison of both techniques for the detection of PVTT. Additionally, there was the possibility that some PVTTs developed between CECT and GA-MRI. To minimize this risk that new PVTTs developed after CECT but before GA-MRI, or vice versa, we only included patients who underwent CECT and GA-MRI within 1 month. Lastly, patients with PVTT detected at CECT or GA-MRI who did not undergo surgery or TACE were excluded 
from our study due to their lack of a reference standard. However, exclusion of those patients may have had an impact on our results, as the diagnostic performance of GA-MRI and CECT would be most clinically important for patients who undergo liver transplantation. Thus, for a more comprehensive assessment of the diagnostic performance of GA-MRI and CECT for the detection of PVTT, inclusion of those patients but with other reference standards, such as contrast-enhanced ultrasound, is warranted in future studies.

In conclusion, GA-MRI was demonstrated to be superior to CECT in terms of sensitivity in the detection of PVTT without significant loss of specificity in patients with HCC meeting the Milan criteria. Therefore, in liver transplantation candidates with HCCs who had previously undergone CECT, GA-MRI should be considered in order to more accurately rule out PVTT, which is a major contraindication to liver transplantation.

\section{Acknowledgment}

The authors appreciate the Medical Research Collaborating Center at the Seoul National University Hospital for their statistical analysis and consultation. The authors also acknowledge Chris Woo, BA, for his editorial expertise.

\section{Statement of Ethics}

This retrospective study was approved by our institutional review board for a chart review, and the requirement for informed consent was waived. This study was conducted ethically in accordance with the World Medical Association Declaration of Helsinki.

\section{Disclosure Statement}

J.S.B. - no relevant relationships; J.M.L.- only activities not related to the present article: institutional grants from RF Medical, Bayer Healthcare, Guerbet, Samsung Medison, Philips Healthcare, GE Healthcare, CMS, and Canon Healthcare; and receipt of payment from Siemens Healthcare, Guerbet, and Bayer Healthcare for lectures, including service on speakers' bureaus; J.H.Y. -: only activities not related to the present article: receipt of payment from Samsung Electronics for consultancy, institutional grant from Bayer, and receipt of payment from Philips Healthcare and Bayer for lectures, including service on speakers' bureaus.; S.J., J.W.C., K.B.L., N.-J.Y., and J.H.L. - no relevant relationships.

\section{Funding Sources}

This study did not receive financial support.

\section{Author Contributions}

Jae Seok Bae was involved in the conception and design, development of methodology, acquisition of data, analysis and interpretation of data, and writing of the manuscript. Jeong Hee Yoon, Siwon Jang, Jin Wook Chung, Kyung Bun Lee, Nam-Joon Yi, and Jeong-Hoon Lee were engaged in the acquisition of data, analysis of data, and review of the manuscript. Jeong Min Lee carried out the conception and design, acquisition of data, analysis and interpretation of data, writing and review of the manuscript, and study supervision.

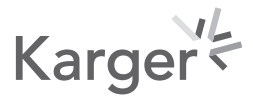




\section{References}

1 Parvey HR, Raval B, Sandler CM. Portal vein thrombosis: imaging findings. AJR Am J Roentgenol. 1994 Jan; 162(1):77-81.

2 Ikai I, Arii S, Kojiro M, Ichida T, Makuuchi M, Matsuyama Y, et al. Reevaluation of prognostic factors for survival after liver resection in patients with hepatocellular carcinoma in a Japanese nationwide survey. Cancer. 2004 Aug;101(4):796-802.

3 Stuart KE, Anand AJ, Jenkins RL. Hepatocellular carcinoma in the United States. Prognostic features, treatment outcome, and survival. Cancer. 1996 Jun;77(11):2217-22.

4 Shah SA, Greig PD, Gallinger S, Cattral MS, Dixon E, Kim RD, et al. Factors associated with early recurrence after resection for hepatocellular carcinoma and outcomes. J Am Coll Surg. 2006 Feb;202(2):275-83.

5 Portolani N, Coniglio A, Ghidoni S, Giovanelli M, Benetti A, Tiberio GA, et al. Early and late recurrence after liver resection for hepatocellular carcinoma: prognostic and therapeutic implications. Ann Surg. 2006 Feb;243(2): 229-35.

6 Piscaglia F, Gianstefani A, Ravaioli M, Golfieri R, Cappelli A, Giampalma E, et al.; Bologna Liver Transplant Group. Criteria for diagnosing benign portal vein thrombosis in the assessment of patients with cirrhosis and hepatocellular carcinoma for liver transplantation. Liver Transpl. 2010 May;16(5):658-67.

7 Qian LJ, Zhu J, Zhuang ZG, Xia Q, Cheng YF, Li JY, et al. Differentiation of neoplastic from bland macroscopic portal vein thrombi using dual-energy spectral CT imaging: a pilot study. Eur Radiol. 2012 Oct;22(10): 2178-85.

8 Yerdel MA, Gunson B, Mirza D, Karayalçin K, Olliff S, Buckels J, et al. Portal vein thrombosis in adults undergoing liver transplantation: risk factors, screening, management, and outcome. Transplantation. 2000 May; 69(9):1873-81.

9 European Association for the Study of the Liver. Electronic address: easloffice@easloffice.eu; European Association for the Study of the Liver. EASL Clinical Practice Guidelines: Management of hepatocellular carcinoma. J Hepatol. 2018 Jul;69(1):182-236.

10 Mitchell DG, Bruix J, Sherman M, Sirlin CB. LI-RADS (Liver Imaging Reporting and Data System): summary, discussion, and consensus of the LI-RADS Management Working Group and future directions. Hepatology. 2015 Mar;61(3):1056-65.

11 Omata M, Lesmana LA, Tateishi R, Chen PJ, Lin SM, Yoshida H, et al. Asian Pacific Association for the Study of the Liver consensus recommendations on hepatocellular carcinoma. Hepatol Int. 2010 Mar;4(2):439-74.

12 Bruix J, Sherman M; American Association for the Study of Liver Diseases. Management of hepatocellular carcinoma: an update. Hepatology. 2011 Mar;53(3):1020-2.

13 European Association for Study of L. European Organisation for R, Treatment of C: EASL-EORTC clinical practice guidelines: management of hepatocellular carcinoma. Eur J Cancer. 2012;48(5):599-641.

14 Kitao A, Zen Y, Matsui O, Gabata T, Kobayashi S, Koda W, et al. Hepatocellular carcinoma: signal intensity at gadoxetic acid-enhanced MR Imaging_correlation with molecular transporters and histopathologic features. Radiology. 2010 Sep;256(3):817-26.

15 Liu X, Zou L, Liu F, Zhou Y, Song B. Gadoxetic acid disodium-enhanced magnetic resonance imaging for the detection of hepatocellular carcinoma: a meta-analysis. PLoS One. 2013 Aug;8(8):e70896.

16 Chen L, Zhang L, Liang M, Bao J, Zhang J, Xia Y, et al. Magnetic resonance imaging with gadoxetic acid disodium for the detection of hepatocellular carcinoma: a meta-analysis of 18 studies. Acad Radiol. 2014 Dec;21(12): 1603-13.

17 Junqiang L, Yinzhong W, Li Z, Shunlin G, Xiaohui W, Yanan Z, et al. Gadoxetic acid disodium (Gd-EOBDTPA)enhanced magnetic resonance imaging for the detection of hepatocellular carcinoma: a meta-analysis. J Magn Reson Imaging. 2014 May;39(5):1079-87.

18 Lee YJ, Lee JM, Lee JS, Lee HY, Park BH, Kim YH, et al. Hepatocellular carcinoma: diagnostic performance of multidetector CT and MR imaging-a systematic review and meta-analysis. Radiology. 2015 Apr;275(1): 97-109.

19 Joo I, Lee JM. Recent Advances in the Imaging Diagnosis of Hepatocellular Carcinoma: Value of Gadoxetic AcidEnhanced MRI. Liver Cancer. 2016 Feb;5(1):67-87.

20 Ahn SS, Kim MJ, Lim JS, Hong HS, Chung YE, Choi JY. Added value of gadoxetic acid-enhanced hepatobiliary phase MR imaging in the diagnosis of hepatocellular carcinoma. Radiology. 2010 May;255(2):459-66.

21 Korean Liver Cancer Association (KLCA); National Cancer Center (NCC). 2018 Korean Liver Cancer Association-National Cancer Center Korea practice guidelines for the management of hepatocellular carcinoma. Korean J Radiol. 2019 Jul;20(7):1042-113.

22 Rohrer M, Bauer H, Mintorovitch J, Requardt M, Weinmann HJ. Comparison of magnetic properties of MRI contrast media solutions at different magnetic field strengths. Invest Radiol. 2005 Nov;40(11):715-24.

23 Feuerlein S, Boll DT, Gupta RT, Ringe KI, Marin D, Merkle EM. Gadoxetate disodium-enhanced hepatic MRI: dose-dependent contrast dynamics of hepatic parenchyma and portal vein. AJR Am J Roentgenol. 2011 Jan; 196(1):W18-24.

24 Seale MK, Catalano OA, Saini S, Hahn PF, Sahani DV. Hepatobiliary-specific MR contrast agents: role in imaging the liver and biliary tree. Radiographics. 2009 0ct;29(6):1725-48.

25 Kim JH, Lee JM, Yoon JH, Lee DH, Lee KB, Han JK, et al. Portal Vein Thrombosis in Patients with Hepatocellular Carcinoma: Diagnostic Accuracy of Gadoxetic Acid-enhanced MR Imaging. Radiology. 2016 Jun;279(3):773-83. 


\begin{tabular}{l|l|}
\hline Liver Cancer 2020;9:293-307 \\
\hline DOI: 10.1159/000505191 & $\begin{array}{l}\text { @ 2020 The Author(s). Published by S. Karger AG, Basel } \\
\text { www.karger.com/lic }\end{array}$ \\
\hline
\end{tabular}

26 Tublin ME, Dodd GD 3rd, Baron RL. Benign and malignant portal vein thrombosis: differentiation by CT characteristics. AJR Am J Roentgenol. 1997 Mar;168(3):719-23.

27 Rossi S, Ghittoni G, Ravetta V, Torello Viera F, Rosa L, Serassi M, et al. Contrast-enhanced ultrasonography and spiral computed tomography in the detection and characterization of portal vein thrombosis complicating hepatocellular carcinoma. Eur Radiol. 2008 Aug;18(8):1749-56.

28 Choi JW, Kim HC, Lee JH, Yu SJ, Kim YJ, Yoon JH, et al. Transarterial chemoembolization of hepatocellular carcinoma with segmental portal vein tumour thrombus. Eur Radiol. 2017 Apr;27(4):1448-58.

29 Okuda K, Musha H, Yoshida T, Kanda Y, Yamazaki T, Jinnouchi S, et al. Demonstration of growing casts of hepatocellular carcinoma in the portal vein by celiac angiography: the thread and streaks sign. Radiology. 1975 Nov;117(2):303-9.

30 Valls C, Pamies JJ, Sancho C, Benasco C, Figueras J, Dominguez J, et al. Computed tomography after Lipiodol chemoembolization in hepatocellular carcinoma. Eur Radiol. 1994;4(3):238-42.

31 Ho VB, Allen SF, Hood MN, Choyke PL. Renal masses: quantitative assessment of enhancement with dynamic MR imaging. Radiology. 2002 Sep;224(3):695-700.

32 Jha RC, Khera SS, Kalaria AD. Portal Vein Thrombosis: Imaging the Spectrum of Disease With an Emphasis on MRI Features. AJR Am J Roentgenol. 2018 Jul;211(1):14-24.

33 Catalano OA, Choy G, Zhu A, Hahn PF, Sahani DV. Differentiation of malignant thrombus from bland thrombus of the portal vein in patients with hepatocellular carcinoma: application of diffusion-weighted MR imaging. Radiology. 2010 Jan;254(1):154-62.

34 Shah ZK, McKernan MG, Hahn PF, Sahani DV. Enhancing and expansile portal vein thrombosis: value in the diagnosis of hepatocellular carcinoma in patients with multiple hepatic lesions. AJR Am J Roentgenol. 2007 May;188(5):1320-3.

35 Rossi S, Rosa L, Ravetta V, Cascina A, Quaretti P, Azzaretti A, et al. Contrast-enhanced versus conventional and color Doppler sonography for the detection of thrombosis of the portal and hepatic venous systems. AJR Am J Roentgenol. 2006 Mar;186(3):763-73.

36 Canellas R, Mehrkhani F, Patino M, Kambadakone A, Sahani D. Characterization of Portal Vein Thrombosis (Neoplastic Versus Bland) on CT Images Using Software-Based Texture Analysis and Thrombus Density (Hounsfield Units). AJR Am J Roentgenol. 2016 Nov;207(5):W81-7.

37 Li J, Li X, Weng J, Lei L, Gong J, Wang J, et al. Gd-EOB-DTPA dynamic contrast-enhanced magnetic resonance imaging is more effective than enhanced 64-slice CT for the detection of small lesions in patients with hepatocellular carcinoma. Medicine (Baltimore). 2018 Dec;97(52):e13964.

38 Lee DH, Lee JM, Baek JH, Shin CI, Han JK, Choi BI. Diagnostic performance of gadoxetic acid-enhanced liver MR imaging in the detection of HCCs and allocation of transplant recipients on the basis of the Milan criteria and UNOS guidelines: correlation with histopathologic findings. Radiology. 2015 Jan;274(1):149-60.

39 Hamm B, Staks T, Mühler A, Bollow M, Taupitz M, Frenzel T, et al. Phase I clinical evaluation of Gd-EOB-DTPA as a hepatobiliary MR contrast agent: safety, pharmacokinetics, and MR imaging. Radiology. 1995 Jun;195(3): 785-92.

40 Vogl TJ, Kümmel S, Hammerstingl R, Schellenbeck M, Schumacher G, Balzer T, et al. Liver tumors: comparison of MR imaging with Gd-EOB-DTPA and Gd-DTPA. Radiology. 1996 Jul;200(1):59-67.

41 Chang KJ, Kamel IR, Macura KJ, Bluemke DA. 3.0-T MR imaging of the abdomen: comparison with 1.5 T. Radiographics. 2008 Nov-Dec;28(7):1983-98.

42 von Falkenhausen MM, Lutterbey G, Morakkabati-Spitz N, Walter O, Gieseke J, Blömer R, et al. High-fieldstrength MR imaging of the liver at 3.0 T: intraindividual comparative study with MR imaging at $1.5 \mathrm{~T}$. Radiology. 2006 Oct;241(1):156-66.

43 Dodd GD 3rd, Memel DS, Baron RL, Eichner L, Santiguida LA. Portal vein thrombosis in patients with cirrhosis: does sonographic detection of intrathrombus flow allow differentiation of benign and malignant thrombus? AJR Am J Roentgenol. 1995 Sep;165(3):573-7.

44 Ricci P, Cantisani V, Biancari F, Drud FM, Coniglio M, Di Filippo A, et al. Contrast-enhanced color Doppler US in malignant portal vein thrombosis. Acta Radiol. 2000 Sep;41(5):470-3.

45 Sherman CB, Behr S, Dodge JL, Roberts JP, Yao FY, Mehta N. Distinguishing tumor from bland portal vein thrombus in liver transplant candidates with hepatocellular carcinoma: The A-VENA criteria. Liver Transpl. 2019 Feb;25(2):207-16.

46 Pettersson H, Ackerman N, Kaude J, Googe RE, Mancuso AA, Scott KN, et al. Gadolinium-DTPA enhancement of experimental soft tissue carcinoma and hemorrhage in magnetic resonance imaging. Acta Radiol. 1987 Jan-Feb;28(1):75-8.

47 Feinstein AR, Cicchetti DV. High agreement but low kappa: I. The problems of two paradoxes. J Clin Epidemiol. 1990;43(6):543-9. 